\title{
Crystal structure of $(3 \mathrm{a} R, 4 R, 5 S, 6 S, 6 \mathrm{a} R)-4,5,6$-triacetoxy-1-benzyl- $3,3 \mathrm{a}, 4,5,6,6 \mathrm{a}-$ hexahydro- $1 \mathrm{H}$ - cyclopent $[c]$-isoxazole, $\mathrm{C}_{19} \mathrm{H}_{23} \mathrm{NO}_{7}$
}

\author{
W. Frey, P. Hilgers, M. Kleban and V. Jäger* \\ Universität Stuttgart. Institut für Organische Chemie, Pfaffenwaldring 55. D-70569 Stuttgart. Germany
}

Received June 28. 2000. CCDC-No. $1267 / 483$

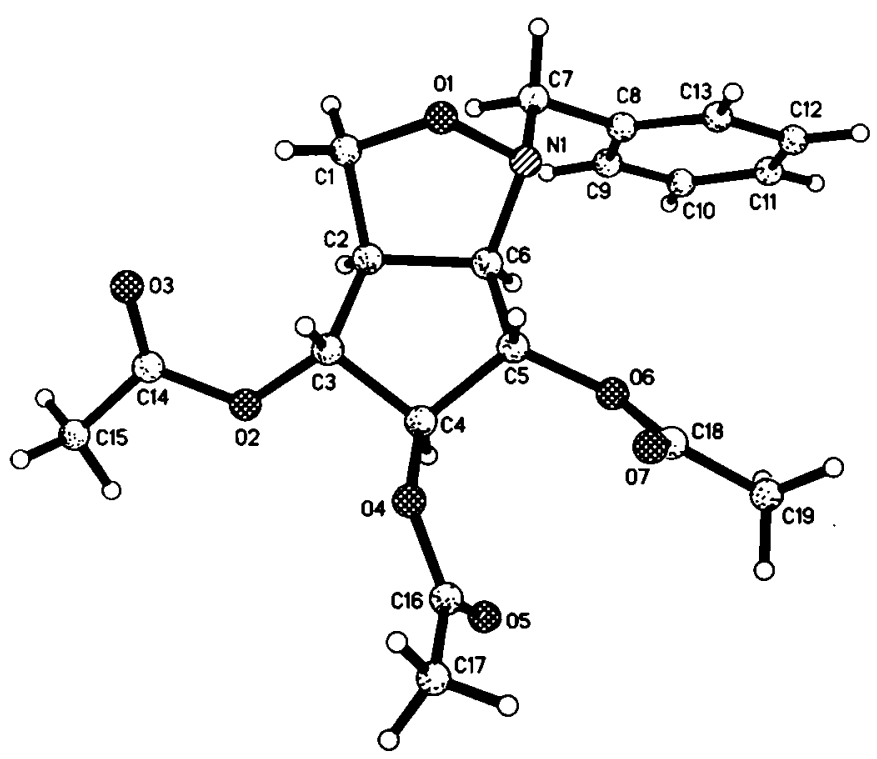

carbons. All large components of the displacement ellipsoids are in-plane with the benzyl moiety, which indicates a strong in-plane vibration of the benzyl moiety. A discrete fixing of disorder positions is not useful, because this is a dynamical effect.

Table 1. Data collection and handling.

Crystal: Wavelength: $\mu$ : Diffractometer, scan mode:
$2 \theta_{\max }$ :

$N(h k l)_{\text {measured, }} N(h k l)_{\text {unique: }}$

Criterion for $I_{\mathrm{obs}}, N(\mathrm{hkl})_{\mathrm{gt}}$ : $N(\text { param })_{\text {refined: }}$

Programs: colorless block, size $0.50 \times 0.65 \times 0.75 \mathrm{~mm}$ Mo $K_{\alpha}$ radiation $(0.71073 \AA)$ $0.98 \mathrm{~cm}^{-1}$

Nicolet P3, Wyckoff $55^{\circ}$

2562,2562

$I_{\text {obs }}>2 \sigma\left(I_{\text {obs }}\right), 1760$

245

SHELXS-86 [6], SHELXL-93 [7]

\section{Abstract}

$\mathrm{C}_{19} \mathrm{H}_{23} \mathrm{NO}_{7}$, orthorhombic, $P 2_{12} 2_{1} 2_{1}$ (No. 19), $a=9.854$ (3) $\AA$, $b=13.353(3) \AA, c=14.949(3) \AA, V=1967.0 \AA^{3}, Z=4$, $R_{\mathrm{gl}}(F)=0.059, w R_{\mathrm{all}}\left(F^{2}\right)=0.145, T=293 \mathrm{~K}$.

\section{Source of material}

The title compound has been obtained by intramolecular nitrone cycloaddition [1]. Starting from 6-deoxy-6-iodo-2,3,4-tri-Oacetyl- $\alpha-D$-glucopyranoside [1-4], reaction with zinc and vita$\min \mathrm{B}_{12}$ as a catalyst afforded the corresponding 5-hexenose [5], which was transformed into the title compound by addition of $\mathrm{N}$-benzyl-hydroxylamine. Purification by flash chromatography on silica and crystallization from methanol gave the isoxazolidine as a single diastereomer in the form of colorless crystals [ $\mathrm{mp}$ $\left.381-383 \mathrm{~K},[\alpha]_{20}^{\mathrm{D}}=-45\left(\mathrm{c}=0.9, \mathrm{CHCl}_{3}\right)\right]$.

\section{Discussion}

The two five-membered rings of the bicyclic isoxazolidine structure each possess a twist-envelope conformation with $\mathrm{O} 1$ and $\mathrm{C4}$ above the respective planes. The hydrogens of the C15-methylgroup show a rotational disorder with six equivalent hydrogen positions. In the benzyl-moiety we found large displacement parameters. They increase from ortho- over meta- to para-
Table 2. Atomic coordinates and displacement parameters (in $\AA^{2}$ ).

\begin{tabular}{|c|c|c|c|c|c|c|}
\hline Atom & Site & Occ. & $x$ & $y$ & $z$ & $U_{\text {iso }}$ \\
\hline$H(1 \mathrm{~A})$ & $4 a$ & & $0.4477(4)$ & $0.1110(4)$ & $0.4683(4)$ & 0.107 \\
\hline $\mathrm{H}(1 \mathrm{~B})$ & $4 a$ & & $0.4620(4)$ & $0.1535(4)$ & $0.5663(4)$ & 0.107 \\
\hline $\mathrm{H}(2)$ & $4 a$ & & $0.2443(4)$ & $0.1877(3)$ & $0.4655(3)$ & 0.073 \\
\hline$H(3)$ & $4 a$ & & $0.2843(4)$ & $0.2116(3)$ & $0.6513(3)$ & 0.067 \\
\hline H(4) & $4 a$ & & $0.0121(4)$ & $0.1975(3)$ & $0.5890(2)$ & 0.061 \\
\hline$H(5)$ & $4 a$ & & $0.1648(3)$ & $0.0385(3)$ & $0.6683(3)$ & 0.061 \\
\hline$H(6)$ & $4 a$ & & $0.1091(4)$ & $0.0519(3)$ & $0.4816(3)$ & 0.066 \\
\hline$H(7 A)$ & $4 a$ & & $0.3802(5)$ & $-0.0986(4)$ & $0.4285(3)$ & 0.106 \\
\hline$H(7 B)$ & $4 a$ & & $0.3145(5)$ & $0.0017(4)$ & $0.3951(3)$ & 0.106 \\
\hline$H(9)$ & $4 a$ & & $0.1346(8)$ & $-0.0157(7)$ & $0.2958(5)$ & 0.163 \\
\hline$H(10)$ & $4 a$ & & $-0.035(1)$ & $-0.106(1)$ & $0.231(1)$ & 0.276 \\
\hline $\mathbf{H}(11)$ & $4 a$ & & $-0.091(2)$ & $-0.257(1)$ & $0.283(1)$ & 0.293 \\
\hline $\mathrm{H}(12)$ & $4 a$ & & $0.0116(8)$ & $-0.3207(7)$ & $0.4084(7)$ & 0.192 \\
\hline$H(13)$ & $4 a$ & & $0.1868(6)$ & $-0.2317(4)$ & $0.4743(4)$ & 0.118 \\
\hline $\mathrm{H}(15 \mathrm{~A})$ & $4 a$ & 0.5 & $0.1721(5)$ & $0.4921(3)$ & $0.5552(3)$ & 0.119 \\
\hline$H(15 B)$ & $4 a$ & 0.5 & $0.3103(5)$ & $0.5097(3)$ & $0.5057(3)$ & 0.119 \\
\hline$H(15 C)$ & $4 a$ & 0.5 & $0.2998(5)$ & $0.5278(3)$ & $0.6090(3)$ & 0.119 \\
\hline$H(15 D)$ & $4 a$ & 0.5 & $0.3493(5)$ & $0.5277(3)$ & $0.5581(3)$ & 0.119 \\
\hline$H(15 E)$ & $4 a$ & 0.5 & $0.2111(5)$ & $0.5100(3)$ & $0.6076(3)$ & 0.119 \\
\hline$H(15 F)$ & $4 a$ & 0.5 & $0.2217(5)$ & $0.4920(3)$ & $0.5042(3)$ & 0.119 \\
\hline$H(17 A)$ & $4 a$ & & $-0.0114(5)$ & $0.2490(4)$ & $0.8655(3)$ & 0.130 \\
\hline$H(17 B)$ & $4 a$ & & $-0.1563(5)$ & $0.2005(4)$ & $0.8639(3)$ & 0.130 \\
\hline$H(17 C)$ & $4 a$ & & $-0.1384(5)$ & $0.3145(4)$ & $0.8417(3)$ & 0.130 \\
\hline$H(19 A)$ & $4 a$ & & $-0.2289(5)$ & $-0.0719(4)$ & $0.6230(3)$ & 0.133 \\
\hline$H(19 B)$ & $4 a$ & & $-0.2713(5)$ & $-0.0383(4)$ & $0.7195(3)$ & 0.133 \\
\hline $\mathrm{H}(19 \mathrm{C})$ & $4 a$ & & $-0.2001(5)$ & $-0.1420(4)$ & $0.7049(3)$ & 0.133 \\
\hline
\end{tabular}

* Correspondence author (e-mail: jager.ioc@po.uni-stuttgart.de) 
Table 3. Atomic coordinates and displacement parameters (in $\AA^{2}$ ).

\begin{tabular}{|c|c|c|c|c|c|c|c|c|c|c|}
\hline Atom & Site & $x$ & $y$ & $z$ & $U_{11}$ & $U_{22}$ & $U_{33}$ & $U_{12}$ & $U_{13}$ & $U_{23}$ \\
\hline$O(1)$ & $4 a$ & $0.3937(3)$ & $0.0126(2)$ & $0.5626(2)$ & $0.056(2)$ & $0.065(2)$ & $0.130(3)$ & $0.008(2)$ & $0.000(2)$ & $-0.003(2)$ \\
\hline$N(1)$ & $4 a$ & $0.2702(3)$ & $-0.0286(3)$ & $0.5250(2)$ & $0.055(2)$ & $0.063(2)$ & $0.082(2)$ & $0.004(2)$ & $0.011(2)$ & $-0.005(2)$ \\
\hline$C(1)$ & $4 a$ & $0.4066(4)$ & $0.1124(4)$ & $0.5273(4)$ & $0.054(2)$ & $0.069(3)$ & $0.146(5)$ & $0.002(2)$ & $0.023(3)$ & $-0.005(3)$ \\
\hline$O(2)$ & $4 a$ & $0.1972(3)$ & $0.3220(2)$ & $0.5805(2)$ & $0.051(1)$ & $0.052(2)$ & $0.081(2)$ & $0.002(1)$ & $0.002(2)$ & $0.003(1)$ \\
\hline $\mathrm{C}(2)$ & $4 a$ & $0.2611(4)$ & $0.1533(3)$ & $0.5223(3)$ & $0.051(2)$ & $0.056(2)$ & $0.076(3)$ & $0.006(2)$ & $0.017(2)$ & $0.005(2)$ \\
\hline$O(3)$ & $4 a$ & $0.4199(3)$ & $0.3490(2)$ & $0.5861(3)$ & $0.053(2)$ & $0.076(2)$ & $0.136(3)$ & $-0.011(2)$ & $0.001(2)$ & $0.016(2)$ \\
\hline$C(3)$ & $4 a$ & $0.2183(4)$ & $0.2180(3)$ & $0.6026(3)$ & $0.047(2)$ & $0.047(2)$ & $0.072(2)$ & $0.003(2)$ & $0.002(2)$ & $0.004(2)$ \\
\hline$O(4)$ & $4 a$ & $0.0498(3)$ & $0.2054(2)$ & $0.7204(2)$ & $0.057(2)$ & $0.062(2)$ & $0.061(2)$ & $0.001(1)$ & $0.001(1)$ & $-0.008(1)$ \\
\hline$C(4)$ & $4 a$ & $0.0830(4)$ & $0.1752(3)$ & $0.6303(2)$ & $0.047(2)$ & $0.054(2)$ & $0.053(2)$ & $0.002(2)$ & $0.002(2)$ & $0.000(2)$ \\
\hline$C(5)$ & $4 a$ & $0.1054(3)$ & $0.0634(3)$ & $0.6206(3)$ & $0.041(2)$ & $0.050(2)$ & $0.060(2)$ & $-0.003(2)$ & $0.003(2)$ & $0.004(2)$ \\
\hline$O(5)$ & $4 a$ & $-0.1685(3)$ & $0.2182(4)$ & $0.6841(3)$ & $0.062(2)$ & $0.216(5)$ & $0.091(2)$ & $0.027(3)$ & $0.007(2)$ & $-0.008(3)$ \\
\hline$O(6)$ & $4 a$ & $-0.0199(2)$ & $0.0078(2)$ & $0.6190(2)$ & $0.056(1)$ & $0.061(2)$ & $0.053(1)$ & $-0.013(1)$ & $0.007(1)$ & $-0.000(1)$ \\
\hline$C(6)$ & $4 a$ & $0.1761(4)$ & $0.0556(3)$ & $0.5299(3)$ & $0.048(2)$ & $0.057(2)$ & $0.060(2)$ & $0.005(2)$ & $0.009(2)$ & $0.002(2)$ \\
\hline$O(7)$ & $4 a$ & $-0.0119(4)$ & $-0.0162(3)$ & $0.7674(2)$ & $0.107(3)$ & $0.132(3)$ & $0.056(2)$ & $-0.046(2)$ & $-0.003(2)$ & $0.013(2)$ \\
\hline$C(7)$ & $4 a$ & $0.2987(5)$ & $-0.0580(4)$ & $0.4305(3)$ & $0.084(3)$ & $0.077(3)$ & $0.103(4)$ & $0.003(3)$ & $0.041(3)$ & $-0.014(3)$ \\
\hline$C(8)$ & $4 a$ & $0.1826(5)$ & $-0.1159(4)$ & $0.3911(3)$ & $0.073(3)$ & $0.098(4)$ & $0.065(3)$ & $0.023(3)$ & $0.020(3)$ & $-0.021(3)$ \\
\hline $\mathrm{C}(9)$ & $4 a$ & $0.1122(8)$ & $-0.0784(7)$ & $0.3185(5)$ & $0.142(6)$ & $0.180(7)$ & $0.085(4)$ & $0.080(6)$ & $0.032(4)$ & $-0.004(5)$ \\
\hline$C(10)$ & $4 a$ & $0.011(1)$ & $-0.132(1)$ & $0.280(1)$ & $0.14(1)$ & $0.43(3)$ & $0.121(8)$ & $0.15(2)$ & $-0.046(9)$ & $-0.12(2)$ \\
\hline$C(11)$ & $4 a$ & $-0.023(2)$ & $-0.221(1)$ & $0.312(1)$ & $0.084(6)$ & $0.39(3)$ & $0.26(2)$ & $0.02(1)$ & $0.000(9)$ & $-0.24(2)$ \\
\hline$C(12)$ & $4 a$ & $0.0387(8)$ & $-0.2594(7)$ & $0.3850(7)$ & $0.110(6)$ & $0.165(7)$ & $0.203(9)$ & $-0.029(6)$ & $0.053(6)$ & $-0.108(8)$ \\
\hline$C(13)$ & $4 a$ & $0.1431(6)$ & $-0.2057(4)$ & $0.4243(4)$ & $0.098(4)$ & $0.091(4)$ & $0.106(4)$ & $-0.009(3)$ & $0.029(3)$ & $-0.027(4)$ \\
\hline$C(14)$ & $4 a$ & $0.3076(5)$ & $0.3805(3)$ & $0.5767(3)$ & $0.064(3)$ & $0.062(3)$ & $0.058(2)$ & $-0.008(2)$ & $0.012(2)$ & $0.003(2)$ \\
\hline$C(15)$ & $4 a$ & $0.2690(5)$ & $0.4871(3)$ & $0.5602(3)$ & $0.089(3)$ & $0.055(3)$ & $0.095(3)$ & $-0.008(2)$ & $0.018(3)$ & $0.005(2)$ \\
\hline$C(16)$ & $4 a$ & $-0.0802(4)$ & $0.2240(4)$ & $0.7390(3)$ & $0.056(3)$ & $0.083(3)$ & $0.071(3)$ & $0.008(2)$ & $0.012(2)$ & $0.001(2)$ \\
\hline$C(17)$ & $4 a$ & $-0.0982(5)$ & $0.2493(4)$ & $0.8363(3)$ & $0.092(4)$ & $0.090(3)$ & $0.077(3)$ & $0.001(3)$ & $0.031(3)$ & $-0.007(3)$ \\
\hline$C(18)$ & $4 a$ & $-0.0683(4)$ & $-0.0250(3)$ & $0.6975(3)$ & $0.070(3)$ & $0.066(3)$ & $0.061(2)$ & $-0.014(2)$ & $0.006(2)$ & $0.001(2)$ \\
\hline$C(19)$ & $4 a$ & $-0.2045(5)$ & $-0.0737(4)$ & $0.6851(3)$ & $0.082(3)$ & $0.105(4)$ & $0.079(3)$ & $-0.034(3)$ & $0.009(3)$ & $0.009(3)$ \\
\hline
\end{tabular}

Acknowledgments. We are grateful to the Landesgraduiertenförderung Baden-Württemberg (doctoral fellowships to P. Hilgers and M. Kleban). For financial support of this work we thank Volkswagen-Stiftung, Hannover and the Fonds der Chemischen Industrie.

\section{References}

1. Hilgers, P.: Synthese und biologische Aktivitat von $\mathrm{N}$-substituierten Amino-hydroxymethyl-cyclopentantriolen der gluco- und manno-Reihe eine neue Klasse von hochwirksamen Glykosidase-Inhibitoren. Dissertation, Universität Stuttgart 2000.

2. Garegg, P. J.; Samuelsson, B.: Novel Reagent System for Converting a Hydroxy-group into an Iodo-group in Carbohydrates with Inversion of Configuration. Part 1. J. Chem. Soc. Chem. Commun. (1979) 978-980.
3. Garegg, P. J.; Samuelsson, B.: Novel Reagent System for Converting a Hydroxy-group into an lodo-group in Carbohydrates with Inversion of Configuration. Part 2. J. Chem. Soc. Perkin Trans. 1 (1980) 2866-2869.

4. Kleban, M.: Diastereoselektive Synthese von Aminocyclopentanpolyolen - Desoxapyranosylamine als neue Klasse von Glykosidase-Inhibitoren. Dissertation, Universität Stuttgart 1996.

5. Kleban, M.; Kautz, U.; Greul, J.; Hilgers, P.; Kugler, R.; Dong, H.-Q.; Jäger, V.: Vitamin $B_{12}$ Catalysis of Zinc-Mediated 6-Deoxy-6-iodopyranoside Fragmentation: A Mild and Convenient Preparation of $\omega-\mathrm{Un}$ saturated Hexose Derivatives (5-Hexenoses). Synthesis (2000) 1027-1033.

6. Sheldrick, G. M.: Phase Annealing in SHELX-90: Direct Methods for Larger Structures. Acta Crystallogr. A46 (1990) 467-473.

7. Sheldrick, G. M.: SHELXL-93, Program for the refinement of crystal structures. University of Göttingen, Germany 1993. 